\title{
The Constitution of Starch
}

$\mathrm{T}$ HE chemical structure of starch has not yet been fully elucidated. In recent years, evidence as to the size of its molecule has been derived from a study of the products obtained when trimethylstarch is broken down; and it is supposed that, like cellulose, starch consists of chains of glucopyranose residues, but united by $\alpha$-glucosidic instead of $\beta$-glucosidic links. Haworth has calculated that the molecule of starch consists of not more than 25-30 glucose units and that these units are associated into physical aggregates of much larger dimensions. On the other hand, Prof. Staudinger has long held the view that the physical properties of starch point to the existence of macro-molecules as distinct from molecular aggregates and in the May issue of the Berichte der deutschen chemischen Gesellschaft he and Herr Husemann adduce fresh evidence in support of this view from a study of the effect of chemical reactions upon the degree of polymerization of various preparations of starch from wheat.

The persistence of a particular degree of poly. merization, after the starch had been regenerated from an acetylated derivative, has been clearly demonstrated and is held to be conclusive proof of the existence of macro-molecules. The reason why such evidence has not hitherto been available is because these macro-molecules are far more sensitive than ordinary molecules to disruptive agencies and it has been shown that even traces of atmospheric oxygen in the solvent exert an enormous effect upon the degree of polymerization.

The starch was first freed from phosphoric acid and purified by repeated precipitation by methanol from solution in formamide. Osmotic pressure measurements of a solution of this in formamide indicated a molecular weight of 286,000 or a polymerization degree of 1770 . Another preparation with a polymerization degree of only 600 was obtained from it by rapid hydrolysis with normal hydrochloric acid. The two preparations were then acetylated, the former giving an insoluble, the latter a soluble, derivative. The acetylated compounds were then hydrolyzed by sodium methylate under very stringent conditions, when it was found that the polymerization of the regenerated starches had only been reduced to 1640 and 530 respectively. In view of the difficulty of eliminating the last traces of atmospheric oxygen during the alkaline hydrolysis, it is claimed that the degree of polymerization of the starch was materially unaltered by the process of acetylation. Viscosity measurements led to the same conclusion.

Starch appears to be not one compound but a kind of polymeric series. From the relation between degree of polymerization and specific viscosity the macromolecules of starch must be quite different in structure from the micelles of cellulose for the viscosity of the latter is 5-10 times as great as that of starch of the same degree of polymerization. Since, however, the viscosity of the solution may be taken as a measure of the length of the dissolved molecules, those of starch must be very much shorter and are therefore coiled. It is also suggested that the coils are branched and the ends of the short glucose chains are linked in glucosidic fashion with hydroxyl groups of other chains. This structure would not only account for a relatively high proportion of tetra. methyl glucose among the fission-products of trimethyl starch but also for the complete absence of aldehydic properties in starch itself.

\section{River Tees Survey}

T $\mathrm{HE}$ beauty and purity of our rivers is a national heritage, and the increasing attention that is being turned towards problems of pollution is therefore welcome. But before the causes and effects of pollution can be truly understood it is necessary to have, as a foundation, a knowledge of the natural, unpolluted state. One of the most important. British contributions in this respect is to be found in a recent publication of the Water Pollution Board of the Department of Scientific and Industrial Research*. This report is concerned with the non-tidal reaches of the River Tees and supplements the work on the tidal region previously published. The survey was undertaken by the Ministry of Agriculture and Fisheries, the research staff being under the direction of Dr. E. S. Russell. The report is drafted by Dr. R. W. Butcher, Dr. J. Longwell and Mr. F. T. K. Pentelow, who were botanist, chemist and zoologist, respectively, on the survey.

A comprehensive chemical and biological survey was made and many experiments performed in the laboratory on the effects of sewage pollution. It was found

- Department of Scientific and Industrial Research. Water Pollution Research Technical Paper No. 6. Survey of the River Tees. Part 3. The Non-Tidal Reaches-Chemical and Biological. By R. W. Butcher, J. Longwell, and F. T. K. Pentelow. (London: H.M. Stationery Office, 1937.) 128. 6 d. net. that the River Tees could conveniently be divided into two regions, namely, from the source at Cross Fell to the junction with the River Skerne at Croft Bridge, a river mileage of 55 miles, and from Croft Bridge to Yarm, a distance of 24 miles. Above the junction with the Skerne, the Tees waters are fairly soft, and slightly alkaline, except at periods of heavy flood. The flora and fauna in this region are uniform, taking into consideration differences to be expected from type of bottom and rate of flow. The only pollution comes from several small sewage works between Middleton-in-Teesdale and Croft, but the dilution by the river is sufficiently great to prevent harmful effects.

The River Skerne is, however, quite different ; its water is very hard and it is heavily polluted with sewage effluent from Darlington. The Skerne water on entering the River Tees produces a marked change in the chemical and biological characteristics of this lower reach. The hardness of the water is increased, as is also the amount of organic matter in solution and in suspension, with a resultant lowering of the oxygen content. The composition of the flora and fauna is different from that above Croft Bridge, but owing to the presence of nutritive substances from the sewage effluent the numbers of plants and animals are greater. By comparing the two regions 